\title{
Acute effect of exercise intensity and duration on acylated ghrelin and hunger in men
}

\author{
David R Broom 1,2, Masashi Miyashita1,3, Lucy K Wasse1,4, Richard Pulsford1,5, \\ James A King', Alice E Thackray' ${ }^{1}$ and David J Stensel'
}

1School of Sport, Exercise and Health Sciences, Loughborough University, Loughborough, UK ${ }^{2}$ Academy of Sport and Physical Activity, Sheffield Hallam University, Sheffield, UK ${ }^{3}$ Faculty of Sport Sciences, Waseda University, Tokorozawa, Japan

${ }^{4}$ Respiratory and Allergy Clinical Research Facility, University Hospital of South Manchester, Manchester, UK ${ }^{5}$ Sport and Health Sciences, University of Exeter, Exeter, UK

\begin{abstract}
Acute exercise transiently suppresses the orexigenic gut hormone acylated ghrelin, but the extent to which exercise intensity and duration determine this response is not fully understood. The effects of manipulating exercise intensity and duration on acylated ghrelin concentrations and hunger were examined in two experiments. In experiment one, nine healthy males completed three, 4-h conditions (control, moderate-intensity running (MOD) and vigorous-intensity running (VIG)), with an energy expenditure of $\sim 2.5 \mathrm{MJ}$ induced in both MOD (55-min running at 52\% peak oxygen uptake $\left(\dot{\mathrm{V}}_{2 \text { peak }}\right)$ ) and VIG (36-min running at $75 \% \dot{V}_{2 \text { peak }}$ ). In experiment two, nine healthy males completed three, 9-h conditions (control, 45-min running (EX45) and 90-min running

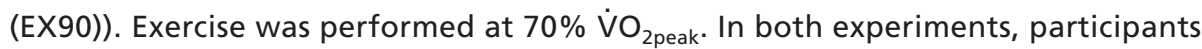
consumed standardised meals, and acylated ghrelin concentrations and hunger were quantified at predetermined intervals. In experiment one, delta acylated ghrelin concentrations were lower than control in MOD $(E S=0.44, P=0.01)$ and VIG $(E S=0.98$, $P<0.001)$; VIG was lower than MOD $(E S=0.54, P=0.003)$. Hunger ratings were similar across the conditions $(P=0.35)$. In experiment two, delta acylated ghrelin concentrations were lower than control in EX45 $(E S=0.77, P<0.001)$ and EX90 $(E S=0.68, P<0.001)$; EX45 and EX90 were similar ( $E S=0.09, P=0.55)$. Hunger ratings were lower than control in EX45 (ES=0.20, $P=0.01$ ) and EX90 (ES=0.27, $P=0.001$ ); EX45 and EX90 were similar $(E S=0.07, P=0.34)$. Hunger and delta acylated ghrelin concentrations remained suppressed at $1.5 \mathrm{~h}$ in EX90 but not EX45. In conclusion, exercise intensity, and to a lesser extent duration, are determinants of the acylated ghrelin response to acute exercise.
\end{abstract}

Correspondence should be addressed to D Stensel

Email

D.J.Stensel@lboro.ac.uk

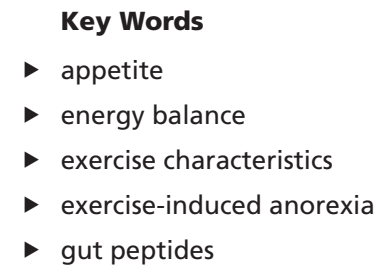

Key Words

appetite

- energy balance

- exercise characteristic

- gut peptides (2017) 232, 411-422

\section{Introduction}

Obesity is characterised by a chronic energy imbalance reflecting a surplus of energy intake above expenditure and remains a major global public health and economic burden (Wang et al. 2011, Ng et al. 2014). Recent years have witnessed significant research into the relationship between exercise, appetite regulation and energy balance (Schubert et al. 2014). Exercise is recommended as a therapeutic weight management strategy because it

Published by Bioscientifica Ltd. 
increases energy expenditure, which contributes to a negative energy balance if unaccompanied by an increase in energy intake (Donnelly et al. 2009). Evidence suggests acute exercise transiently suppresses feelings of hunger during and shortly after exercise (Broom et al. 2007, 2009, King et al. 2010a), which has been termed 'exerciseinduced anorexia' (King et al. 1994). Furthermore, these responses often coincide with exercise-induced fluctuations in hormones that regulate energy balance and appetite (Schubert et al. 2014).

Appetite and energy intake are regulated by the neuroendocrine system, of which gut peptides play an integral role as episodic signals for hunger and satiety (Karra \& Batterham 2010, Hussain \& Bloom 2013). Ghrelin is the only known orexigenic gut peptide and is predominantly secreted from the stomach (Karra \& Batterham 2010). Ghrelin exists in two forms (acylated and unacylated) and although only $10-20 \%$ of circulating ghrelin is acylated, it is believed that this form is solely responsible for appetite stimulation (Ghigo et al. 2005). Considering the central role of acylated ghrelin in appetite regulation, it is not surprising that the interaction between exercise and acylated ghrelin continues to attract scientific enquiry.

Acute moderate- to high-intensity exercise suppresses acylated ghrelin concentrations (King et al. 2013, Schubert et al. 2014). This hormonal alteration appears transient and typically coincides with a reduction in hunger during and immediately after exercise (Broom et al. 2007, 2009, King et al. 2010a). Exercise intensity has been identified as a potential determinant modulating the acylated ghrelin response to exercise (Broom et al. 2007, King et al. 2010a), with suppression occurring after exercise at higher $\left(\geq 60 \%\right.$ peak oxygen uptake $\left.\left(\dot{\mathrm{V}}_{2 \text { peak }}\right)\right)$ (Broom et al. 2007, 2009, King et al. 2010a) but not lower ( $\leq 50 \% \dot{V}_{2 \text { peak }}$ ) (Ueda et al. 2009, King et al. 2010b) intensities. Studies comparing acute moderate- vs highintensity exercise suggest exercising at a higher intensity may be more potent for suppressing acylated ghrelin concentrations (Deighton et al. 2013, Metcalfe et al. 2015). However, the effect of isoenergetic exercise bouts at different intensities has revealed contrasting findings (Sim et al. 2014, Martins et al. 2015, Howe et al. 2016); therefore, further research is required to elucidate the importance of exercise intensity on appetite regulation.

Alterations in ghrelin concentrations and hunger perceptions may also be influenced by manipulations in exercise duration. Erdmann et al. (2007) reported that 30,60 and $120 \mathrm{~min}$ of cycling at $50 \mathrm{~W}$ resulted in a similar increase in total ghrelin concentrations
$(50-70 \mathrm{pg} / \mathrm{mL})$ during exercise without any changes in hunger. However, the assessment of total ghrelin may obscure important changes in acylated ghrelin (Hosoda et al. 2004), and exercise studies measuring total ghrelin have yielded equivocal findings (King et al. 2013). The effect of exercise duration on acylated ghrelin concentrations has not yet been examined and may have important implications regarding the use of exercise as a weight control strategy.

This investigation comprises two experiments that aimed to advance the understanding of appetite and hormonal responses to different acute exercise manipulations. Experiment one compared the effect of acute isoenergetic moderate- and vigorous-intensity running on acylated ghrelin concentrations and hunger perceptions. In experiment two, the acylated ghrelin and hunger responses to single bouts of 45- and 90-min running were examined.

\section{Methods}

\section{Participants}

This investigation contains two experimental studies that were approved by the University Ethical Advisory Committee. Two different groups of healthy, recreationally active men provided their written informed consent to participate in one of the experiments. Information from a health screen questionnaire revealed that all participants were metabolically healthy, non-smokers, not taking medication, body mass stable for at least 6 months $( \pm 2 \mathrm{~kg})$ and not currently dieting. Physical and physiological characteristics of participants are presented in Table 1.

\section{Preliminary measures}

Participants attended the laboratory for two preliminary visits before the main conditions in each experiment. During the first visit, anthropometric data (stature, body mass, waist circumference and skinfold thickness) were collected, and participants were familiarised with exercising on the treadmill (RUNRACE, Techno gym, Gambettola, Italy).

During the second visit, participants completed two exercise tests. The first test consisted of a 16-min submaximal incremental running test to determine the relationship between running speed and oxygen consumption. Participants completed $4 \times 4$-min stages with the initial running speed set between 7 and $8 \mathrm{~km} / \mathrm{h}$

Published by Bioscientifica Ltd 
Table 1 Physical and physiological characteristics in experiments one and two.

\begin{tabular}{|c|c|c|}
\hline Characteristic & $\begin{array}{l}\text { Experiment one } \\
\qquad(n=9)\end{array}$ & $\begin{array}{c}\text { Experiment two } \\
\qquad(n=9)\end{array}$ \\
\hline Age (years) & $21.4(1.7)$ & $23.2(2.1)$ \\
\hline Body mass $(\mathrm{kg})$ & $78.3(11.0)$ & $72.0(5.6)$ \\
\hline Stature $(m)$ & $1.79(0.07)$ & $1.78(0.05)$ \\
\hline Body mass index $\left(\mathrm{kg} / \mathrm{m}^{2}\right)$ & $24.5(2.4)$ & $22.7(1.5)$ \\
\hline Sum of skinfolds (mm) & $33.1(5.7)$ & $26.1(4.5)$ \\
\hline Percent body fat (\%) & $15.3(2.7)$ & $12.0(2.3)$ \\
\hline Waist circumference $(\mathrm{cm})$ & $77.7(5.7)$ & $76.7(2.1)$ \\
\hline $\begin{array}{l}\text { Peak oxygen uptake } \\
(\mathrm{mL} / \mathrm{kg} / \mathrm{min})\end{array}$ & $58(6)$ & $63(6)$ \\
\hline
\end{tabular}

Values are mean (s.D.).

depending on the participant's fitness level, which was increased by $1-1.5 \mathrm{~km} / \mathrm{h}$ at the start of each subsequent stage. Oxygen consumption and carbon dioxide production were determined from expired air samples collected in the final minute of each stage along with the participant's rating of perceived exertion (RPE) using Borg's 6-20 scale (Borg 1973). Heart rate was monitored continuously using short-range telemetry (Polar A3, Kempele, Finland).

After 30-min standardised rest, $\dot{\mathrm{V}} \mathrm{O}_{2 \text { peak }}$ was measured using an incremental uphill treadmill protocol at a constant speed (Taylor et al. 1955). The initial treadmill gradient was set at 3.5\%, which was increased by $2.5 \%$ every $3 \mathrm{~min}$ until volitional exhaustion (Taylor et al. 1955). Peak oxygen consumption was determined from an expired air sample collected during the final minute of the test when participants indicated that they could only continue for an additional $1 \mathrm{~min}$. Heart rate and RPE were monitored throughout the test as described previously. Data from the two preliminary exercise tests were used to determine the running speeds required during the main conditions.

\section{Experimental design}

In each experiment, participants completed three, 1-day conditions in a random order separated by at least one week. Participants weighed, recorded and replicated their food intake in the $24 \mathrm{~h}$ before each main condition. Participants abstained from caffeine, alcohol and strenuous physical activity during the same period. All conditions commenced between 08:00 and 09:00 after an overnight fast of at least $10 \mathrm{~h}$. The study design in both experiments is presented in Fig. 1.

\section{Experiment one: exercise intensity}

Nine men (20-25 years) completed three, 4-h experimental conditions: control, moderate-intensity
Experiment one: exercise intensity

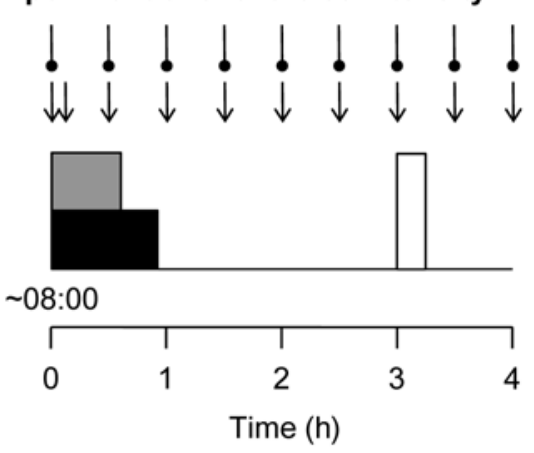

Key:

Hunger rating

Venous blood sample

Standardised meal

Vigorous-intensity exercise

Moderate-intensity exercise

西 45 min exercise

90 min exercise

Experiment two: exercise duration
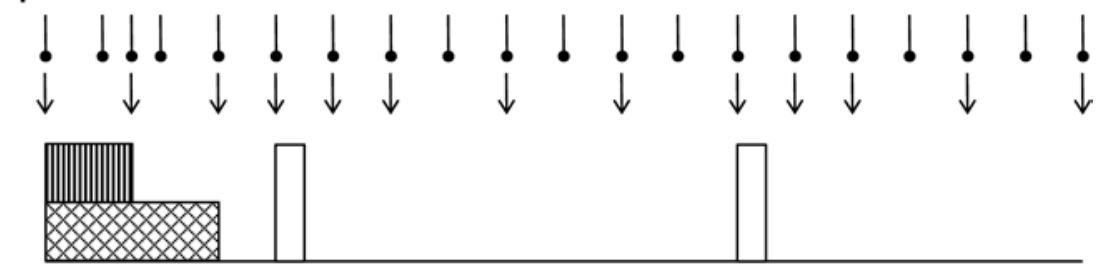

$\sim 08: 00$

\begin{tabular}{|c|c|c|c|c|c|c|c|c|}
\hline$\Gamma$ & $T$ & $T$ & $T$ & $T$ & $T$ & $T$ & $T$ & $T$ \\
\hline 0 & 1 & 2 & 3 & 4 & 5 & 6 & 7 & 8 \\
\hline
\end{tabular}

Time (h)

Figure 1

Schematic representation of the study protocol in experiment one and two. http://joe.endocrinology-journals.org DOI: $10.1530 / \mathrm{JOE}-16-0561$
(C) 2017 Society for Endocrinology Printed in Great Britain 
running (MOD) and vigorous-intensity running (VIG). Participants rested in the laboratory throughout the control condition. The exercise conditions commenced with participants running on the treadmill at a speed predicted to elicit either $50 \% \dot{\mathrm{V}} \mathrm{O}_{2 \text { peak }}$ (MOD) or $75 \%$ $\dot{\mathrm{V}} \mathrm{O}_{2 \text { peak }}$ (VIG), which was designed to induce a gross energy expenditure of $2510 \mathrm{~kJ}$. Expired air samples were collected at regular intervals to calculate the relative exercise intensity, and the treadmill speed was adjusted occasionally to ensure that the target intensity was met. The exercise energy expenditure and substrate oxidation were estimated via indirect calorimetry (Frayn 1983). Heart rate was monitored throughout, and RPE was recorded during the last $10 \mathrm{~s}$ of each expired air sampling period. After the exercise bout, participants rested in the laboratory for the remainder of the condition.

A standardised meal prescribed relative to body mass was provided at $3 \mathrm{~h}$ and consumed within $15 \mathrm{~min}$, which consisted of white bread, tuna, mayonnaise, chocolate bar, potato crisps, apple and orange juice. The standardised meal provided $60 \mathrm{~kJ}$ energy, $2.13 \mathrm{~g}$ (56\% of meal total energy) carbohydrate, $0.53 \mathrm{~g}$ (15\%) protein and $0.47 \mathrm{~g}$ (29\%) fat per kilogram body mass. Water was provided ad libitum throughout each condition.

\section{Experiment two: exercise duration}

Nine men (21-28 years) completed three, 9-h experimental conditions: control, 45-min running (EX45) and 90-min running (EX90). Participants rested in the laboratory throughout the control condition. During the exercise conditions, participants ran on the treadmill at a speed predicted to elicit $70 \% \dot{\mathrm{VO}}_{2 \text { peak }}$ for $45 \mathrm{~min}$ (EX45) or $90 \mathrm{~min}$ (EX90). Expired air samples were collected at regular intervals to calculate the relative exercise intensity, and the treadmill speed was adjusted occasionally to ensure that the target intensity was achieved. The exercise energy expenditure and substrate oxidation were estimated via indirect calorimetry (Frayn 1983). Heart rate was monitored throughout, and RPE was recorded during the last $10 \mathrm{~s}$ of each expired air sampling period. After the exercise bout, participants rested in the laboratory for the remainder of the condition.

Participants consumed identical standardised meals prescribed relative to body mass within $15 \mathrm{~min}$ at 2 and $6 \mathrm{~h}$. The meals consisted of white bread, Cheddar cheese, mayonnaise, butter, potato crisps, milkshake powder and whole milk. The standardised meals provided $46 \mathrm{~kJ}$ energy, $0.95 \mathrm{~g}$ (33\%) carbohydrate,
$0.31 \mathrm{~g}(11 \%)$ protein and $0.69 \mathrm{~g}(56 \%)$ fat per kilogram body mass. Water was provided ad libitum throughout each condition.

\section{Hunger perceptions}

Ratings of perceived hunger were assessed at baseline (fasted) and every $30 \mathrm{~min}$ during both experiments using a $100 \mathrm{~mm}$ Visual Analogue Scale (Flint et al. 2000). An additional measurement was taken at $45 \mathrm{~min}$ in experiment two.

\section{Blood sampling}

Venous blood samples were collected via a cannula (Venflon, Becton Dickinson) inserted into an antecubital vein. All samples were collected in the semisupine position, except the samples scheduled during exercise, which were taken while participants straddled the treadmill. Plasma acylated ghrelin concentrations were determined from blood samples collected into pre-chilled $4.9 \mathrm{~mL}$ EDTA monovettes (Sarsedt, Leicester, UK) at 0 (baseline), 0.08, 0.5, 1, 3, 3.5 and $4 \mathrm{~h}$ in experiment one and at 0 (baseline), 0.75, 1.5, 2, 3, 6, 7 and $9 \mathrm{~h}$ in experiment two. These monovettes contained p-hydroxymercuribenzoic acid (PHMB) to prevent the degradation of acylated ghrelin by protease. Monovettes were spun at $1287 \boldsymbol{g}$ for $10 \mathrm{~min}$ at $4^{\circ} \mathrm{C}$ (Burkard, Hertfordshire, UK). The plasma supernatant was aliquoted into a storage tube, and $100 \mu \mathrm{L}$ of $1 \mathrm{M}$ hydrochloric acid was added per millilitre of plasma (Hosoda et al. 2004). Samples were re-centrifuged at $1287 \mathrm{~g}$ for $5 \mathrm{~min}$ at $4^{\circ} \mathrm{C}$ prior to storage at $-80^{\circ} \mathrm{C}$ for later analysis.

Plasma glucose and insulin concentrations were determined from blood samples collected into pre-chilled $9 \mathrm{~mL}$ EDTA monovettes (Sarsedt, Leicester, UK). Glucose concentrations were measured at 0 (baseline), 0.5, 1, 1.5, $2,2.5,3,3.5$ and $4 \mathrm{~h}$ in experiment one and at 0 (baseline), $0.75,1.5,2,2.5,3,4,5,6,6.5,7,8$ and $9 \mathrm{~h}$ in experiment 2 . Insulin concentrations were measured at 0 (baseline), 0.5 , $1,2,3,3.5$ and $4 \mathrm{~h}$ in experiment one and at 0 (baseline), $0.75,1.5,2,3,6,7$ and $9 \mathrm{~h}$ in experiment two. Monovettes were centrifuged immediately at $1681 \mathrm{~g}$ for $10 \mathrm{~min}$ at $4^{\circ} \mathrm{C}$ (Burkard, Hertfordshire, UK). The plasma supernatant was aliquoted into Eppendorf tubes prior to storage at $-80^{\circ} \mathrm{C}$ for subsequent analysis.

At each blood sampling point, haemoglobin concentration (via the cyanmethaemoglobin method) and haematocrit (via microcentrifugation) were

Published by Bioscientifica Ltd. 
determined to estimate acute changes in plasma volume (Dill \& Costill 1974).

\section{Biochemical analysis}

In both experiments, plasma acylated ghrelin concentrations were determined using a commercially available enzyme immunoassay (SPI BIO, Montigny le Bretonneaux, France). Plasma glucose concentrations were determined using an automated centrifugal analyser (Cobas Mira Plus, Roche). For experiment one, plasma insulin concentrations were determined by a solid phase ${ }^{125}$ I radioimmunoassay available in a commercial kit (MP Biomedicals, Orangeburg, NY, USA) using an automated gamma counter system (Cobra II, Packard Instrument, Downers Grove, IL, USA). For experiment two, plasma insulin concentrations were quantified using a commercially available enzymelinked immunoassay (Mercodia, Uppsala, Sweden). The within-batch coefficient of variation for acylated ghrelin, glucose and insulin were 7.0, 1.4 and 8.9\%, respectively, in experiment one and 2.2, 0.6 and $4.7 \%$, respectively, in experiment two.

\section{Statistical analyses}

Data were analysed using IBM Statistics Software for Windows, version 21 (IBM Corporation). Time-averaged area under the curve (AUC) values were calculated using the trapezoidal rule. Normality of the data was checked using ShapiroWilk tests. Normally distributed data are presented as mean (s.D.). Data for hunger, glucose and insulin were natural $\log$ transformed prior to analysis. These data are presented as geometric mean (95\% confidence interval), and analysis is based on ratios of the geometric means. Acylated ghrelin concentrations are presented relative to baseline concentrations (i.e., delta) to minimise the potential influence of dayto-day biological variation in this appetite hormone (Deighton et al. 2014).

In both experiments, linear mixed models repeated for condition were used to examine differences in exercise responses, fasting (baseline) concentrations and AUC values. Differences in metabolite concentrations between conditions over time were examined using linear mixed models repeated for condition and time. In experiment two, temporal changes in AUC responses for hunger and acylated ghrelin between experimental conditions were examined over sub-sections of the 9-h measurement period $(0-2,2-6$ and 6-9h) using separate linear mixed models with condition as the sole factor. All linear mixed models included a random effect for each participant. Where significant condition and interaction effects were found, post hoc analysis was performed using the Holm-Bonferroni correction for multiple comparisons (Atkinson 2002). Correction of acylated ghrelin, glucose and insulin concentrations for changes in plasma volume did not alter the interpretation of the results; therefore, the unadjusted values are presented for simplicity. Pearson's product moment correlations were used to examine relationships between variables. Statistical significance was accepted as $P<0.05$. Absolute standardised effect sizes (ES) are included to supplement important findings. An ES of 0.2 was considered the minimum important difference in all outcome measures, 0.5 moderate and 0.8 large (Cohen 1988). Graphical representations of results

Table 2 Responses to treadmill running in experiments one and two.

\begin{tabular}{|c|c|c|c|}
\hline & \multicolumn{3}{|c|}{ Experiment one: exercise intensity } \\
\hline & Moderate intensity & Vigorous intensity & Effect size \\
\hline Exercise time (min) & $55(7)$ & $36(5)$ & $2.97^{a}$ \\
\hline Treadmill speed $(\mathrm{km} / \mathrm{h})$ & $7.5(0.8)$ & $11.0(1.5)$ & $2.84^{a}$ \\
\hline Heart rate (beats/min) & $136(15)$ & $163(19)$ & $1.57^{a}$ \\
\hline Rating of perceived exertion & $12(1)$ & $14(2)$ & $1.49 a$ \\
\hline Oxygen uptake (L/min) & $2.37(0.35)$ & $3.41(0.40)$ & $2.74^{a}$ \\
\hline Percent peak oxygen uptake (\%) & $52(3)$ & $75(4)$ & $6.32^{\mathrm{a}}$ \\
\hline Respiratory exchange ratio & $0.90(0.03)$ & $0.96(0.04)$ & $1.95^{\mathrm{a}}$ \\
\hline Fat oxidation (\%) & $32(9)$ & $11(10)$ & $2.26^{a}$ \\
\hline Carbohydrate oxidation (\%) & $68(9)$ & $89(10)$ & $2.26^{a}$ \\
\hline Gross energy expenditure (kJ) & $2580(152)$ & $2504(165)$ & 0.48 \\
\hline
\end{tabular}

\begin{tabular}{|c|c|c|}
\hline \multicolumn{3}{|c|}{ Experiment two: exercise duration } \\
\hline $45 \mathrm{~min}$ & $90 \mathrm{~min}$ & Effect size \\
\hline $45(0)$ & $90(0)$ & - \\
\hline $10.6(1.4)$ & $10.4(1.3)$ & 0.09 \\
\hline $169(11)$ & $169(12)$ & 0.00 \\
\hline $13(1)$ & $14(1)$ & 0.59 \\
\hline $3.19(0.36)$ & $3.17(0.34)$ & 0.06 \\
\hline $70(2)$ & $70(2)$ & 0.20 \\
\hline $0.93(0.05)$ & $0.89(0.11)$ & 0.52 \\
\hline $24(10)$ & $33(27)$ & 0.44 \\
\hline $76(10)$ & $67(27)$ & 0.44 \\
\hline $2918(329)$ & $5949(653)$ & $5.86^{b}$ \\
\hline
\end{tabular}

Values are mean (s.D.).

aSignificant difference between moderate-intensity exercise and vigorous-intensity exercise $(P<0.05)$; bsignificant difference between 45 -min exercise and 90-min exercise $(P<0.05)$.

http://joe.endocrinology-journals.org DOI: $10.1530 / \mathrm{JOE}-16-0561$ (c) 2017 Society for Endocrinology Printed in Great Britain
Published by Bioscientifica Ltd. 
Table 3 Fasting and time-averaged total area under the concentration vs time curve in the control, moderate-intensity exercise and vigorous-intensity exercise conditions in experiment one.

\begin{tabular}{|c|c|c|c|c|}
\hline & Control & Moderate-intensity exercise & Vigorous-intensity exercise & Main effect condition $P$ \\
\hline \multicolumn{5}{|l|}{ Hunger } \\
\hline Fasting (mm) & $33(18-60)$ & $30(16-55)$ & $25(14-46)$ & 0.50 \\
\hline TAUC $(\mathrm{mm})$ & $53(44-65)$ & $49(41-60)$ & $51(42-61)$ & 0.65 \\
\hline \multicolumn{5}{|l|}{ Acylated ghrelin } \\
\hline Fasting $(\mathrm{pg} / \mathrm{mL})$ & $67.2(31.4)$ & $68.1(25.9)$ & $78.9(42.0)$ & 0.57 \\
\hline Delta TAUC (pg/mL) & $2.29(8.21)$ & $-6.83(11.76)$ & $-17.78(19.16)$ & $0.01^{a}$ \\
\hline \multicolumn{5}{|l|}{ Glucose } \\
\hline Fasting (mmol/L) & $5.21(4.68-5.80)$ & $5.44(4.89-6.07)$ & $5.52(4.96-6.15)$ & 0.63 \\
\hline TAUC (mmol/L) & $5.20(4.87-5.54)$ & $5.22(4.89-5.57)$ & $5.52(5.17-5.89)$ & 0.06 \\
\hline \multicolumn{5}{|l|}{ Insulin } \\
\hline Fasting (pmol/L) & $137(107-175)$ & 175 (137-224) & $168(131-215)$ & 0.19 \\
\hline TAUC (pmol/L) & $297(238-371)$ & $292(234-365)$ & $302(242-377)$ & 0.95 \\
\hline
\end{tabular}

Values for acylated ghrelin are mean (SD) for $n=8$. Values for hunger, glucose and insulin are geometric mean (95\% confidence interval) for $n=9$, and statistical analyses are based on natural log transformed data.

aSignificant difference between vigorous-intensity exercise and control conditions (linear mixed model $P<0.05$ after Holm-Bonferroni correction). TAUC, time-averaged total area under the concentration vs time curve.

are presented as mean (s.e.m.) to avoid distortion of the figures.

\section{Results}

\section{Experiment one: exercise intensity}

Exercise responses Exercise responses for MOD and VIG are shown in Table 2. Exercise duration was significantly shorter, and treadmill speed, heart rate, RPE and oxygen uptake were all greater in VIG compared with MOD $(P \leq 0.05)$. Respiratory exchange ratio was higher in VIG than MOD $(P<0.001)$. The relative contribution of carbohydrate and fat oxidation to energy provision was higher and lower, respectively, in VIG compared with MOD (both $P<0.001$ ). Gross energy expenditure was not significantly different between the exercise conditions $(P=0.38)$.

Hunger perceptions Fasting hunger ratings were similar across the conditions at baseline $(P=0.50)$ (Table 3$)$. Linear mixed models revealed no differences in hunger ratings across the conditions (main effect condition $P=0.35$; main effect time $P<0.001$; condition by time interaction $P=0.78$ ) (Fig. 2).
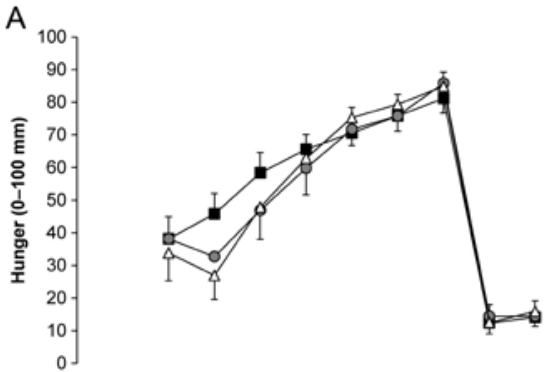

B

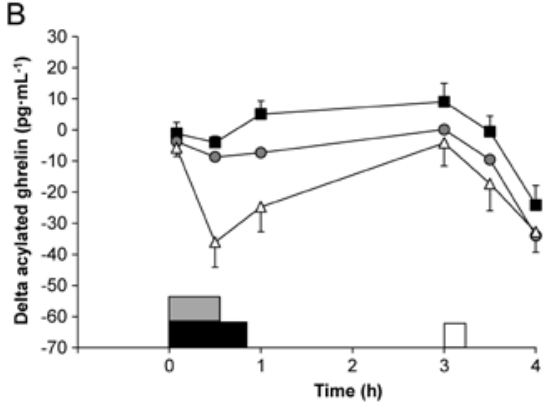

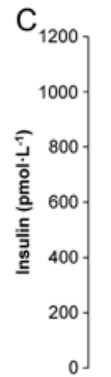

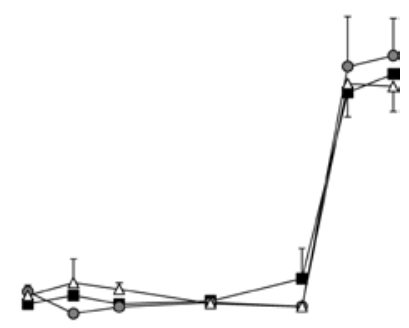

D

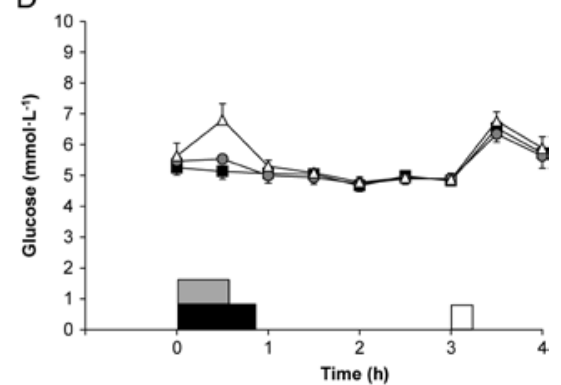

Figure 2

Perceptions of hunger $(A)$ and concentrations of plasma delta acylated ghrelin (B), insulin (C) and glucose (D) in the control ( $\square)$, moderate-intensity exercise $(O)$ and vigorous-intensity exercise $(\triangle)$ conditions. Values are mean (SEM), $n=9$ for hunger, insulin and glucose and $n=8$ for acylated ghrelin. Black rectangle indicates moderateintensity exercise, grey rectangle indicates vigorous-intensity exercise and open rectangle indicates consumption of the standardised meal. 
Hunger total AUC was similar across the conditions $(P=0.65)$ (Table 3).

\section{Acylated ghrelin, glucose and insulin} concentrations Boxplot analysis of acylated ghrelin total AUC values identified one participant as an outlier (Field 2009). This participant exhibited a mean acylated ghrelin concentration 21 times greater than the mean SD of the remaining participants (range: $74-1489 \mathrm{pg} / \mathrm{mL}$ ). Therefore, this participant was removed, and results are presented for eight participants. Fasting acylated ghrelin concentrations were similar across the conditions at baseline $(P=0.57)$ (Table 3). Linear mixed models for delta acylated ghrelin revealed a significant main effect of condition $(P<0.001)$, time $(P<0.001)$ and condition by time interaction $(P=0.03)$ (Fig. 2$)$. Post hoc analysis of between-condition differences revealed that delta acylated ghrelin concentrations were lower than control in MOD (ES $=0.44, P=0.01)$ and VIG (ES $=0.98, P<0.001)$; VIG was lower than MOD (ES=0.54, $P=0.003$ ). Post hoc analysis of the condition by time interaction revealed that the delta acylated ghrelin concentration was lower than control in VIG at $0.5 \mathrm{~h}(\mathrm{ES}=5.49, P=0.005)$ and $1 \mathrm{~h}(\mathrm{ES}=2.46$, $P=0.02)$; VIG was lower than MOD at $0.5 \mathrm{~h}(\mathrm{ES}=4.68$, $P=0.02$ ). Delta total AUC for acylated ghrelin was lower in VIG compared with control (ES=2.45, $P=0.01$ ) (Table 3).

Fasting glucose concentrations were similar across the conditions at baseline $(P=0.63)$ (Table 3). Linear mixed models for glucose identified a main effect of condition $(P=0.02)$ and time $(P<0.001)$, but not a condition by time interaction $(P=0.46)$ (Fig. 2). Post hoc analysis of between-condition differences revealed that mean VIG glucose concentration was $6 \%$ and $5 \%$ higher than control (ES=0.31, $P=0.02$ ) and MOD (ES=0.27, $P=0.04$ ), respectively; control and MOD were similar $(1 \%$; $\mathrm{ES}=0.04$, $P=0.73$ ). The VIG glucose AUC was meaningfully, albeit not significantly, higher than control $(6 \%$; $E S=0.62$, $P=0.09$ ) and $\mathrm{MOD}(6 \%$; ES $=0.58, P=0.09)$; control and MOD were not different ( $0 \%$; ES $=0.05, P=0.86$ ) (Table 3).

Fasting insulin concentrations were similar across the conditions at baseline $(P=0.19)$ (Table 3). No differences in insulin concentrations were seen across the conditions (main effect condition $P=0.28$; main effect time $P<0.001$; condition by time interaction $P=0.26$ ) (Fig. 2). Insulin total AUC was similar across the conditions $(P=0.95)$ (Table 3$)$.

Correlations There were no significant correlations between delta acylated ghrelin concentrations and changes in hunger, glucose or insulin values.

\section{Experiment two: exercise duration}

Exercise responses Exercise responses for EX45 and EX90 are displayed in Table 2. The only significant difference was the anticipated increase in gross energy expenditure for EX90 compared with EX45 $(P<0.001)$.

Hunger perceptions Fasting hunger ratings were similar across the conditions at baseline $(P=0.73)$ (Table 4$)$. Linear mixed models for hunger revealed a significant main effect of condition $(P=0.001)$, time $(P<0.001)$ and condition by time interaction $(P<0.001)$ (Fig. 3).

Table 4 Fasting and time-averaged total area under the concentration vs time curve in the control, 45-min exercise and 90-min exercise conditions in experiment two.

\begin{tabular}{|c|c|c|c|c|}
\hline & Control & 45-min exercise & 90-min exercise & Main effect condition $P$ \\
\hline \multicolumn{5}{|l|}{ Hunger } \\
\hline Fasting (mm) & $45(30-68)$ & $47(31-72)$ & $43(28-65)$ & 0.73 \\
\hline TAUC $(\mathrm{mm})$ & $37(27-49)$ & $31(23-42)$ & $30(22-40)$ & $0.02^{a}$ \\
\hline \multicolumn{5}{|l|}{ Acylated ghrelin } \\
\hline Fasting $(\mathrm{pg} / \mathrm{mL})$ & $159(140)$ & $163(140)$ & $153(128)$ & 0.88 \\
\hline Delta TAUC (pg/mL) & $-7.44(48.30)$ & $-55.20(77.34)$ & $-46.56(53.75)$ & 0.07 \\
\hline \multicolumn{5}{|l|}{ Glucose } \\
\hline Fasting (mmol/L) & $5.04(4.78-5.32)$ & $5.06(4.80-5.34)$ & $5.04(4.78-5.32)$ & 0.98 \\
\hline TAUC (mmol/L) & $5.05(4.88-5.23)$ & $5.32(5.14-5.50)$ & $5.35(5.17-5.54)$ & 0.06 \\
\hline \multicolumn{5}{|l|}{ Insulin } \\
\hline Fasting (pmo/L) & $21.3(12.1-37.7)$ & $19.6(11.1-34.6)$ & $17.1(9.7-30.2)$ & 0.74 \\
\hline TAUC (pmol/L) & $67.8(47.7-96.5)$ & $68.2(47.9-97.1)$ & $61.5(43.2-87.5)$ & 0.81 \\
\hline
\end{tabular}

Values for acylated ghrelin are mean (SD) for $n=9$. Values for hunger, glucose and insulin are geometric mean (95\% confidence interval) for $n=9$, and statistical analyses are based on natural log transformed data.

aSignificant difference between 90-min exercise and control conditions (linear mixed model $P<0.05$ after Holm-Bonferroni correction).

TAUC, time-averaged total area under the concentration vs time curve.

http://joe.endocrinology-journals.org DOI: 10.1530/JOE-16-0561
๑ 2017 Society for Endocrinology Printed in Great Britain
Published by Bioscientifica Ltd 
A
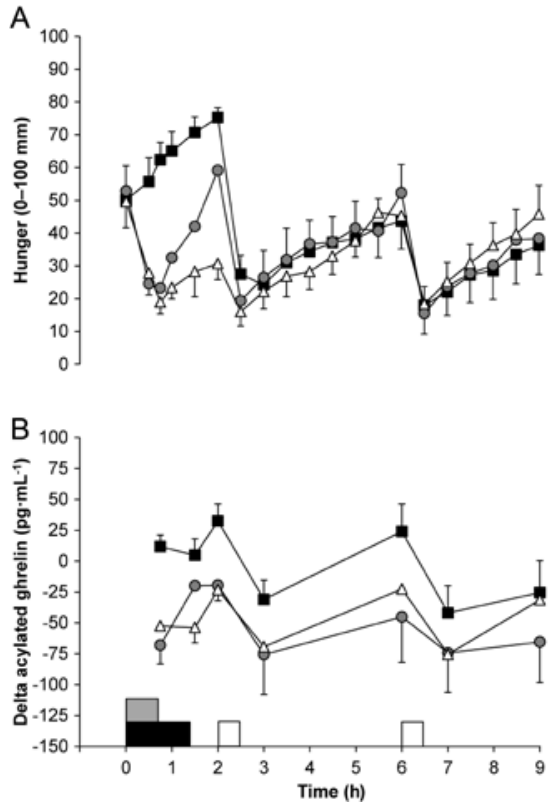

C

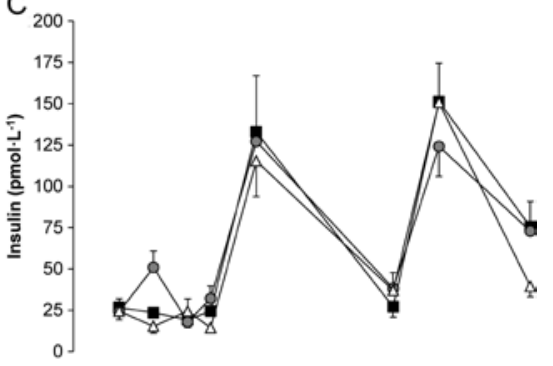

D

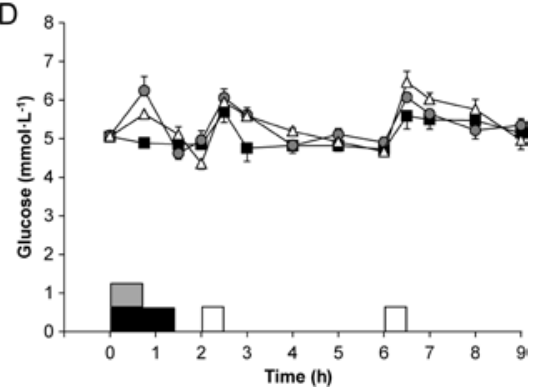

Figure 3

Perceptions of hunger (A), and concentrations of plasma delta acylated ghrelin (B), insulin (C) and glucose (D) in the control $(\square), 45$-min exercise (O) and 90 -min exercise $(\triangle)$ conditions. Values are mean (SEM), $n=9$ for hunger, acylated ghrelin, insulin and glucose. Black rectangle indicates 90-min exercise, grey rectangle indicates 45 -min exercise and open rectangles indicates consumption of the standardised meals.
Post hoc analysis of between-condition differences revealed that hunger perceptions were $15 \%$ and $20 \%$ lower than control in EX45 (ES=0.20, $P=0.01)$ and EX90 (ES=0.27, $P=0.001)$, respectively; EX45 and EX90 were similar ( $-6 \%$; ES=0.07, $P=0.34)$. Post hoc analysis of the condition by time interaction revealed that hunger perceptions were lower than control in EX45 at 0.5, 0.75 and $1 \mathrm{~h}$ (all ES $\geq 1.71, P \leq 0.05$ ); EX90 was lower than control at $0.5,0.75,1,1.5$ and $2 \mathrm{~h}$ (all ES $\geq 1.30, P \leq 0.05$ ). The hunger total AUC was 14\% and 18\% lower than control in EX45 (ES=0.36, $P=0.07)$ and EX90 $(\mathrm{ES}=0.48$, $P=0.02)$, respectively; EX45 and EX90 were similar $(-5 \%$; $\mathrm{ES}=0.13, P=0.42$ ) (Table 4). Specifically, hunger AUC was lower than control between 0 and $2 \mathrm{~h}$ in EX45 $(-43 \%$; $\mathrm{ES}=1.96, P=0.001)$ and EX90 (-54\%; ES=2.77, $P<0.001)$; EX90 was meaningfully, albeit not significantly, lower than EX45 (-20\%; ES=0.81, $P=0.08)$.

\section{Acylated ghrelin, glucose and insulin} concentrations Fasting acylated ghrelin concentrations were similar across the conditions at baseline $(P=0.88) \quad($ Table 4). Linear mixed models for delta acylated ghrelin identified a significant main effect for condition $(P<0.001)$ and time $(P<0.001)$, but not a condition by time interaction $(P=0.47)$ (Fig. 3). Post hoc analysis of between-condition differences revealed that delta acylated ghrelin concentrations were lower than control in EX45 (ES=0.77, $P<0.001)$ and EX90 $(\mathrm{ES}=0.68$, $P<0.001)$; EX45 and EX90 were similar $(\mathrm{ES}=0.09$, $P=0.55)$. The delta total AUC for acylated ghrelin was lower than control in $\mathrm{EX} 45(\mathrm{ES}=0.99, P=0.03)$ and
EX90 (ES=0.81, $P=0.07)$, respectively; EX45 and EX90 were similar $(\mathrm{ES}=0.18, P=0.68)$ (Table 4). Specifically, EX45 was lower than control between 0 and $2 \mathrm{~h}(\mathrm{ES}=1.93$, $P<0.001)$ and 2 and $6 \mathrm{~h}(\mathrm{ES}=1.05, P=0.05)$; EX90 was lower than control between 0 and $2 \mathrm{~h}(\mathrm{ES}=2.16, P<0.001)$ and 2 and $6 \mathrm{~h}(\mathrm{ES}=0.83, P=0.18)$.

Fasting glucose concentrations were similar across the conditions at baseline $(P=0.98)$ (Table 4$)$. Linear mixed models for glucose identified a significant main effect for condition $(P<0.001)$, time $(P<0.001)$ and condition by time interaction $(P<0.001)$ (Fig. 3). Post hoc analysis of between-condition differences revealed that mean glucose concentrations were 5\% higher than control in EX45 $(\mathrm{ES}=0.40, P=0.001)$ and EX90 $(\mathrm{ES}=0.40, P=0.001)$; EX45 and EX90 were similar $(0 \%$; ES=0.00, $P=0.97)$. Post hoc analysis of the condition by time interaction revealed the glucose concentration was higher than control in EX45 at $0.75 \mathrm{~h}(26 \% ; \mathrm{ES}=4.17, P=0.01)$. Linear mixed models identified a trend for differences in glucose total AUC across the conditions $(P=0.06)$, but post hoc analysis revealed no significant between-condition differences after Holm-Bonferroni correction ( $P \geq 0.09)$ (Table 4).

Fasting insulin concentrations were similar across the conditions at baseline $(P=0.74)$ (Table 4$)$. Linear mixed models for insulin revealed a significant main effect for condition $(P=0.03)$ and time $(P<0.001)$, but not a condition by time interaction $(P=0.18)$ (Fig. 3). Post hoc analysis of between-condition differences revealed mean insulin concentrations were 20\% and 25\% lower in EX90 than control $(\mathrm{ES}=0.22, P=0.08)$ and $\mathrm{EX} 45 \quad(\mathrm{ES}=0.27$, $P=0.03)$, respectively; $\mathrm{CON}$ and $\mathrm{EX} 45$ were similar

Published by Bioscientifica Ltd. 
(6\%; $\mathrm{ES}=0.05, P=0.61)$. Insulin total AUC was not significantly different across the conditions $(P=0.81)$ (Table 4).

Correlations There were no significant correlations between delta acylated ghrelin concentrations and changes in hunger, glucose or insulin values for any time period.

\section{Discussion}

The purpose of the present experiments was to elucidate the effect of exercise intensity and duration on acylated ghrelin concentrations and hunger perceptions. The primary findings are that isoenergetic vigorous-intensity running transiently suppressed acylated ghrelin concentrations to a greater extent than moderateintensity running, but was not accompanied by a change in hunger. Furthermore, acylated ghrelin concentrations and hunger were suppressed to a similar extent during 45- and 90-min treadmill running, but the effect appears prolonged when the exercise duration is extended.

Research has demonstrated that acute exercise suppresses acylated ghrelin concentrations, with perturbations returning to control values within $30 \mathrm{~min}$ after exercise (King et al. 2013, Schubert et al. 2014). Experiment one extends these findings by demonstrating that acylated ghrelin concentrations were reduced to a greater extent during vigorous-intensity running than moderate-intensity running, despite a similar exercise-induced energy expenditure. This is consistent with previous research identifying exercise intensity as an important determinant of the acylated ghrelin response to acute exercise, with suppression occurring at intensities $\geq 60 \% \quad \dot{\mathrm{V}} \mathrm{O}_{2 \text { peak }}$ typically (Broom et al. 2007, 2009, Ueda et al. 2009, King et al. 2010a,b). The importance of exercise intensity is highlighted further by studies reporting that sprint interval exercise suppresses acylated ghrelin to a greater extent than moderateintensity exercise (Deighton et al. 2013, Metcalfe et al. 2015). However, studies directly comparing isoenergetic bouts of moderate- and vigorous- to high-intensity exercise have reported contrasting findings, with one study reporting greater suppression of acylated ghrelin at the higher exercise intensity (akin to experiment one) (Sim et al. 2014), whereas others demonstrate a similar level of suppression independent of exercise intensity (Martins et al. 2015, Howe et al. 2016). The discrepancy in findings is likely related to key variations in the protocols adopted including differences in the participant groups, exercise energy expenditure, completion of exercise in the fasted or postprandial state and the timing of meal intake. Differences in meal size and macronutrient composition and methods utilised to quantify acylated ghrelin are likely to further confound the interpretation of these findings. Additional work is clearly required to elucidate the impact of exercise intensity on acylated ghrelin.

Surprisingly, despite the decrease in acylated ghrelin during vigorous-intensity exercise, hunger did not differ significantly between conditions. Although this contrasts previous studies reporting simultaneous reductions in acylated ghrelin and hunger in response to exercise (Broom et al. 2007, 2009, King et al. 2010a), exerciseinduced changes in acylated ghrelin and hunger do not always occur in parallel (Deighton et al. 2013, Sim et al. 2014, Martins et al. 2015). This apparent disassociation highlights the complex nature of appetite regulation, which involves the interaction of many physiological and psychological factors (Hussain \& Bloom 2013).

In accordance with previous studies, experiment two demonstrated a reduction in acylated ghrelin concentrations and hunger in both exercise conditions (Broom et al. 2007, 2009, King et al. 2010a). Although the hunger and acylated ghrelin responses were not statistically different between the two exercise interventions, the values remained suppressed at $1.5 \mathrm{~h}$ in the 90 -min, but not 45 -min, exercise bout (Fig. 3). This suggests that increasing the exercise duration may extend the exercise-induced suppression in hunger and acylated ghrelin concentrations. Although this is the first study to investigate the effect of exercise duration on acylated ghrelin concentrations, Erdmann et al. (2007) reported no differences in total ghrelin concentrations in response to 30-, 60- and 120-min cycling. However, acylated ghrelin is the form of ghrelin thought to be solely responsible for appetite stimulation (Ghigo et al. 2005) and may be obscured when total ghrelin is measured (Hosoda et al. 2004). Furthermore, research examining the effect of acute exercise on total ghrelin concentrations has yielded equivocal findings with evidence of acute increases, decreases and no change (King et al. 2013).

Similar to experiment one, there was a divergence in the acylated ghrelin and hunger responses to acute exercise, further highlighting the complexity of appetite regulation. Although simultaneous reductions in acylated ghrelin and hunger were seen during exercise, hunger ratings returned to similar values between conditions at $2.5 \mathrm{~h}$, but acylated ghrelin remained suppressed in the exercise conditions after meal consumption. The reason for this disparity is unclear, but the findings of the present

Published by Bioscientifica Ltd 
experiments contribute to the debate concerning the importance of reductions in acylated ghrelin as a potential determinant of hunger.

The physiological significance of transient reductions in acylated ghrelin during and after exercise is not fully understood. The divergence between acylated ghrelin and hunger demonstrated in the present experiments and previous studies (Deighton et al. 2013, Sim et al. 2014, Martins et al. 2015) challenges the role that acylated ghrelin plays in mediating appetite responses to exercise. Furthermore, although the implementation of standardised meals in the present experiments precluded the assessment of energy intake, the consensus of evidence suggests that acute aerobic exercise does not stimulate compensatory increases in appetite and energy intake on the same day (Deighton \& Stensel 2014). This may point to the existence of alternative compensatory mechanisms; for example, reductions in unstructured physical activity (i.e., non-exercise activity thermogenesis) and/or increased sedentary behaviours on the day of exercise, but further work is required to support this. Nevertheless, acylated ghrelin is the only gut peptide known to stimulate appetite and energy intake, with circulating concentrations increasing preprandially and decreasing postprandially on a meal-to-meal basis (Cummings et al. 2004). Consequently, this temporal pattern of fluctuation in acylated ghrelin is indicative of an important role in coordinating meal initiation and/or termination (Cummings et al. 2004, Karra \& Batterham 2010).

The mechanisms underpinning the transient exercise-induced suppression of acylated ghrelin are unclear but are likely to reflect processes interfering with the synthesis and/or secretion of acylated ghrelin into the circulation. A recent review suggests that the redistribution of blood flow from splanchnic areas to active skeletal muscle may be particularly pertinent for suppressing ghrelin and appears to be dependent on the exercise intensity (Hazell et al. 2016). Exerciseinduced changes in glucose and insulin concentrations have also been implicated mechanistically (Hazell et al. 2016), with elevations associated with decreased ghrelin concentrations (Flanagan et al. 2003, Cummings \& Overduin 2007, Iwakura et al. 2015). The elevation in glucose concentration during vigorous-intensity exercise in experiment one and both exercise conditions in experiment two coincided with the reduction in acylated ghrelin concentrations. However, insulin concentrations were reduced in the 90-min exercise condition, and previous exercise studies provide conflicting findings by reporting no effect of glucose and insulin on acylated ghrelin concentrations (Broom et al. 2007, 2009). Further research is required to develop a mechanistic understanding of the exercise-induced suppression of acylated ghrelin.

One limitation of the present experiments represents the measurement of a single appetiteregulating hormone. Despite the unique role of acylated ghrelin as the only appetite-stimulating gut hormone, it is only one component of the appetiteregulating neuroendocrine system. Therefore, it may be prudent for future studies to investigate anorexigenic hormones (e.g., peptide-YY, glucagon-like peptide-1, pancreatic polypeptide and cholecystokinin) to provide a broader scientific understanding of the role exercise intensity and duration play in modulating appetite regulation. Secondly, appetite perceptions were limited to the assessment of hunger; however, utilising multiple scales (e.g., satisfaction, fullness and prospective food consumption) may provide a more holistic insight into appetite perceptions (Blundell et al. 2010). Finally, we recruited a small group of healthy and recreationally active men to both experiments, which may limit applications to other population groups and the ability to detect meaningful associations between variables. Additional research is needed in overweight and obese populations who are most likely to benefit from weight management strategies. Despite these limitations, our findings provide important insight into the role that exercise intensity and duration play in modulating hormonal and hunger responses to exercise.

In conclusion, the present experiments demonstrate that exercise intensity, and to a lesser extent, duration, are determinants of the acylated ghrelin response to exercise. Acylated ghrelin is transiently suppressed after a bout of exercise, an effect that appears greater when exercise is performed at a higher intensity. Increasing the exercise duration may prolong the transient suppression in hunger and acylated ghrelin, but the disassociation between hunger and acylated ghrelin responses requires further investigation. Future research is warranted to examine these responses chronically and in overweight/ obese populations for whom exercise may be a therapeutic strategy for weight management.

\section{Declaration of interest}

The authors declare that there is no conflict of interest that could be perceived as prejudicing the impartiality of the research reported.

Published by Bioscientifica Ltd 


\section{Funding}

The research was supported by the National Institute for Health Research (NIHR) Diet, Lifestyle and Physical Activity Biomedical Research Unit at University Hospitals of Leicester and Loughborough University. The views expressed are those of the authors and not necessarily those of the NHS, the NIHR or the Department of Health.

\section{Acknowledgments}

The authors thank the volunteers for their participation in this study.

\section{References}

Atkinson G 2002 Analysis of repeated measurements in physical therapy research: multiple comparisons amongst level means and multifactorial designs. Physical Therapy in Sport 3 191-203. (doi:10.1054/ ptsp.2002.0123)

Blundell J, de Graaf C, Hulshof T, Jebb S, Livingstone B, Lluch A, Mela D, Salah S, Schuring E, van der Knaap H, et al. 2010 Appetite control: methodological aspects of the evaluation of foods. Obesity Reviews $\mathbf{1 1}$ 251-270. (doi:10.1111/j.1467-789X.2010.00714.x)

Borg GA 1973 Perceived exertion: a note on 'history' and methods. Medicine and Science in Sports 5 90-93. (doi:10.1249/00005768197300520-00017)

Broom DR, Stensel DJ, Bishop NC, Burns SF \& Miyashita M 2007 Exerciseinduced suppression of acylated ghrelin in humans. Journal of Applied Physiology 102 2165-2171. (doi:10.1152/japplphysiol.00759.2006)

Broom DR, Batterham RL, King JA \& Stensel DJ 2009 Influence of resistance and aerobic exercise on hunger, circulating levels of acylated ghrelin, and peptide YY in healthy males. American Journal of Physiology: Regulatory Integrative and Comparative Physiology 296 R29-R35. (DOI:10.1152/ajpregu.90706.2008)

Cohen J 1988 Statistical Power Analysis for the Behavioural Sciences, edn 2, pp 22-25. Hillsdale, NJ, USA: Lawrence Erlbaum Associates.

Cummings DE, Frayo RS, Marmonier C, Aubert R \& Chapelot D 2004 Plasma ghrelin levels and hunger scores in humans initiating meals voluntarily without time- and food-related cues. American Journal of Physiology: Endocrinology and Metabolism 287 E297-E304. (DOI:10.1152/ajpendo.00582.2003)

Cummings DE \& Overduin J 2007 Gastrointestinal regulation of food intake. Journal of Clinical Investigation 117 13-23. (doi:10.1172/ JCI30227)

Deighton K, Barry R, Connon CE \& Stensel DJ 2013 Appetite, gut hormone and energy intake responses to low volume sprint interval and traditional endurance exercise. European Journal of Applied Physiology 113 1147-1156. (doi:10.1007/s00421-012-2535-1)

Deighton K \& Stensel DJ 2014 Creating an acute energy deficit without stimulating compensatory increases in appetite: is there an optimal exercise protocol? Proceedings of the Nutrition Society 73 352-358. (doi:10.1017/S002966511400007X)

Deighton K, Batterham RL \& Stensel DJ 2014 Appetite and gut peptide responses to exercise and calorie restriction. The effect of modest energy deficits. Appetite 81 52-59. (doi:10.1016/j.appet.2014.06.003)

Dill DB \& Costill DL 1974 Calculation of percentage changes in volumes of blood, plasma, and red cells in dehydration. Journal of Applied Physiology 37 247-248.

Donnelly JE, Blair SN, Jakicic JM, Manore MM, Rankin JW \& Smith BK 2009 American College of Sports Medicine Position Stand.

Appropriate physical activity intervention strategies for weight loss and prevention of weight regain for adults. Medicine and Science in Sports and Exercise 41 459-471. (doi:10.1249/MSS.0b013e3181949333)

Erdmann J, Tahbaz R, Lippl F, Wagenpfeil S \& Schusdziarra V 2007 Plasma ghrelin levels during exercise - effects of intensity and duration. Regulatory Peptides 143 127-135. (doi:10.1016/j.regpep.2007.05.002)
Field A 2009 Discovering Statistics using SPSS, edn 3. London, UK: Sage. Flanagan DE, Evans ML, Monsod TP, Rife F, Heptulla RA, Tamborlane WV \& Sherwin RS 2003 The influence of insulin on circulating ghrelin. American Journal of Physiology: Endocrinology and Metabolism 284 E313-E316. (DOI:10.1152/ajpendo.00569.2001)

Flint A, Raben A, Blundell JE \& Astrup A 2000 Reproducibility, power and validity of visual analogue scales in assessment of appetite sensations in single test meal studies. International Journal of Obesity 24 38-48. (doi:10.1038/sj.ijo.0801083)

Frayn KN 1983 Calculation of substrate oxidation rates in vivo from gaseous exchange. Journal of Applied Physiology: Respiratory, Environmental and Exercise Physiology 55 628-634.

Ghigo E, Broglio F, Arvat E, Maccario M, Papotti M \& Muccioli G 2005 Ghrelin: more than a natural GH secretagogue and/or an orexigenic factor. Clinical Endocrinology 62 1-17. (doi:10.1111/j.13652265.2004.02160.x)

Hazell TJ, Islam H, Townsend LK, Schmale MS \& Copeland JL 2016 Effects of exercise intensity on plasma concentrations of appetiteregulating hormones: potential mechanisms. Appetite 98 80-88. (doi:10.1016/j.appet.2015.12.016)

Hosoda H, Doi K, Nagaya N, Okumura H, Nakagawa E, Enomoto M, Ono F \& Kangawa K 2004 Optimum collection and storage conditions for ghrelin measurements: octanoyl modification of ghrelin is rapidly hydrolyzed to desacyl ghrelin in blood samples. Clinical Chemistry 50 1077-1080. (doi:10.1373/ clinchem.2003.025841)

Howe SM, Hand TM, Larson-Meyer DE, Austin KJ, Alexander BM \& Manore MM 2016 No effect of exercise intensity on appetite in highly-trained endurance women. Nutrients 8 E223. (doi:10.3390/ nu8040223)

Hussain SS \& Bloom SR 2013 The regulation of food intake by the gutbrain axis: implications for obesity. International Journal of Obesity $\mathbf{3 7}$ 625-633. (doi:10.1038/ijo.2012.93)

Iwakura H, Kangawa K \& Nakao K 2015 The regulation of circulating ghrelin - with recent updates from cell-based assays. Endocrine Journal 62 107-122. (doi:10.1507/endocrj.EJ14-0419)

Karra E \& Batterham RL 2010 The role of gut hormones in the regulation of body weight and energy homeostasis. Molecular and Cellular Endocrinology 316 120-128. (doi:10.1016/j.mce.2009.06.010)

King NA, Burley VJ \& Blundell JE 1994 Exercise-induced suppression of appetite: effects on food intake and implications for energy balance. European Journal of Clinical Nutrition 48 715-724.

King JA, Miyashita M, Wasse LK \& Stensel DJ 2010a Influence of prolonged treadmill running on appetite, energy intake and circulating concentrations of acylated ghrelin. Appetite 54 492-498. (doi:10.1016/j.appet.2010.02.002)

King JA, Wasse LK, Broom DR \& Stensel DJ $2010 b$ Influence of brisk walking on appetite, energy intake, and plasma acylated ghrelin. Medicine and Science in Sports and Exercise 42 485-492. (doi:10.1249/ MSS.0b013e3181ba10c4)

King JA, Wasse LK, Stensel DJ \& Nimmo MA 2013 Exercise and ghrelin. A narrative overview of research. Appetite 68 83-91. (doi:10.1016/j. appet.2013.04.018)

Martins C, Stensvold D, Finlayson G, Holst J, Wisloff U, Kulseng B, Morgan L \& King NA 2015 Effect of moderate- and highintensity acute exercise on appetite in obese individuals. Medicine and Science in Sports and Exercise $\mathbf{4 7}$ 40-48. (doi:10.1249/ MSS.0000000000000372)

Metcalfe RS, Koumanov F, Ruffino JS, Stokes KA, Holman GD, Thompson D \& Vollaard NBJ 2015 Physiological and molecular responses to an acute bout of reduced-exertion high-intensity interval training (REHIT). European Journal of Applied Physiology 115 2321-2334. (doi:10.1007/s00421-015-3217-6)

$\mathrm{Ng}$ M, Fleming T, Robinson M, Thomson B, Graetz N, Margono C, Mullany EC, Biryukov S, Abbafati C, Abera SF, et al. 2014 Global, regional, and national prevalence of overweight and obesity in children and adults during 1980-2013: a systematic analysis for

Published by Bioscientifica Ltd 
the Global Burden of Disease Study 2013. Lancet 384 766-781. (doi:10.1016/S0140-6736(14)60460-8)

Schubert MM, Sabapathy S, Leveritt M \& Desbrow B 2014 Acute exercise and hormones related to appetite regulation: a metaanalysis. Sports Medicine 44 387-403. (doi:10.1007/s40279-0130120-3)

Sim AY, Wallman KE, Fairchild TJ \& Guelfi KJ 2014 High-intensity intermittent exercise attenuates ad-libitum energy intake. International Journal of Obesity 38 417-422. (doi:10.1038/ ijo.2013.102)
Taylor HL, Buskirk E \& Henschel A 1955 Maximal oxygen intake as an objective measure of cardio-respiratory performance. Journal of Applied Physiology 8 73-80.

Ueda SY, Yoshikawa T, Katsura Y, Usui T, Nakao H \& Fujimoto S 2009 Changes in gut hormone levels and negative energy balance during aerobic exercise in obese young males. Journal of Endocrinology 201 151-159. (doi:10.1677/JOE-08-0500)

Wang YC, McPherson K, Marsh T, Gortmaker SL \& Brown M 2011 Health and economic burden of the projected obesity trends in the USA and the UK. Lancet 378 815-825. (doi:10.1016/S0140-6736(11)60814-3)

Received in final form 4 November 2016

Accepted 13 December 2016

Accepted Preprint published online 20 December 2016
Published by Bioscientifica Ltd. 